\title{
Die Pandemie trifft alle, aber nicht alle gleich. Ungleichheiten in Bezug auf Gesundheit und Care
}

\author{
Katharina Heimerl, Elisabeth Reitinger, Barbara Pichler
}

\section{Einleitung}

Soziale Ungleichheit existiert nicht erst seit Beginn der COVID-19-Pandemie. Wir wissen, dass der Wohlstand in dieser Welt ungleich verteilt ist, und dass diese Ungleichheit weiterhin zunimmt. Der Wirtschaftswissenschaftler Joseph Stiglitz argumentiert in seinen weithin rezipierten Büchern, dass diese Ungleichheit auf bewussten Entscheidungen beruht sie ist das Ergebnis von ungerechter Politik und fehlgeleiteten Prioritäten (Stiglitz 2012, 2016).

Als Wissenschaftlerinnen beschäftigen wir uns mit Gesundheit und mit Care und beobachten, dass die ungleiche Verteilung von ökonomischen Ressourcen weitreichende Konsequenzen hat, unter anderem auf die Gesundheit der Menschen und auf die Verteilung von bezahlter und unbezahlter Care-Arbeit zwischen den Geschlechtern. Sozioökonomische, gesundheitliche und care-bezogene Ungleichheiten verstärken einander auch und gerade unter Bedingungen von COVID-19. Sowohl die gesundheitlichen Auswirkungen der Infektion als auch die Maßnahmen zur Eindämmung des Virus verstärken die sozioökonomischen Ungleichheiten und umgekehrt: ärmere Menschen und Länder sind stärker von der Pandemie betroffen (WHO Europe 2020), dies wollen wir in der Folge aufzeigen.

Kümpers und Alisch (2018) fassen soziale Ungleichheit einerseits als vertikale Ungleichheit, welche mit Konzepten von Klassen und Schichten beschrieben wird und Hierarchien in der Gesellschaft wiedergibt. Indikatoren dafür sind Einkommen, Vermögen, formaler Bildungsstatus und beruflicher Status. Andererseits benennen sie Ungleichheiten entlang horizontaler Merkmale, die sich nicht rein aus der sozialen Position erklären lassen. Dazu zählen Ungleichheiten aufgrund von Geschlecht oder ethnischer Zugehörigkeit und körperliche Ungleichheiten.

Das Zusammenwirken von horizontalen und vertikalen Merkmalen sozialer Ungleichheit und damit verbundener ungleicher Lebens- und 
Lebensverwirklichungschancen wird als ,Intersektionalität' bezeichnet (Kümpers/Alisch 2018, 53f).

Der Begriff der Intersektionalität geht auf den US-amerikanischen Black Feminism der 1980er Jahre zurück. Die Juristin Kimberlé Crenshaw (2013) bediente die Metapher der Straßenkreuzung, um auf der Basis von Diskriminierungserfahrungen schwarzer Frauen zu veranschaulichen, dass Diskriminierungen aus unterschiedlichen Richtungen kommen können und zu sehr spezifischen Verletzungen bzw. Verletzungsrisiken an bestimmten Kreuzungspunkten führen. Wie Lutz et al. (2013) bezüglich Intersektionalität treffend anmerken, ist es unmöglich, alle Ungleichheitsdimensionen bzw. Differenzkategorien umfassend zu repräsentieren. Dennoch, so die Autorinnen, hat Intersektionalität das Potential, fortwährend für neue mögliche Auslassungen sensibel zu bleiben.

Das Ziel dieses Beitrages ist es, Ungleichheiten aufzuzeigen, die unsere Forschungsschwerpunkte im Bereich von Palliative Care, Gerontologie und Dementia Care sowie Feministischer Care-Ethik betreffen. Mit einer „intersektionalen Forschungshaltung“ (Bolte/Lahn 2015, 62), deren Ziel es ist, ungleiche Machtverhältnisse, Exklusion und Marginalisierung zu verringern, möchten wir uns der Frage annähern, inwiefern durch die Pandemie in Bezug auf Gesundheit und Care Ungleichheiten erzeugt, tradiert oder zugespitzt werden. Eingangs ist uns wichtig darzustellen, dass Gesundheit und Care-Arbeit auch schon vor der Corona-Pandemie ungleich verteilt waren, um im Anschluss Wechselwirkungen zwischen Pandemie und Ungleichheiten im Bereich Gesundheit und Care aufzuzeigen.

\section{Gesundheit war schon vor der Pandemie ungleich verteilt}

Gesundheit bedeutet für uns umfassendes körperliches, seelisches und soziales Wohlbefinden.

Grundlegende Bedingungen und konstituierende Momente von Gesundheit sind Frieden, angemessene Wohnbedingungen, Bildung, Ernährung, Einkommen, ein stabiles Öko-System, eine sorgfältige Verwendung vorhandener Naturressourcen, soziale Gerechtigkeit und Chancengleichheit (WHO 1986).

Diese Formulierung der Ottawa-Charta für Gesundheitsförderung der WHO (1986) ist für uns von ungebrochener Aktualität. Sie macht deutlich, dass die Chancen, ein gesundes Leben zu führen, ungleich verteilt sind 
und es daher entsprechende Maßnahmen und Strukturen braucht, um Ungerechtigkeiten auszugleichen.

Der sozioökonomische Status wird regelmäßig als zentraler Einflussfaktor auf Gesundheit und Krankheit sowohl in Hinblick auf Morbidität (Inzidenz und Prävalenz) als auch auf Lebenserwartung, d. h. die Mortalität, beschrieben. Mit großer Stabilität in allen untersuchten Ländern der westlichen Welt sowie in verschiedenen Zeiträumen können diese Unterschiede nachgewiesen werden (Stronegger 2007). Benachteiligte sozioökonomische Positionen, die v. a. durch Einkommen, Bildung und berufliche Situation charakterisiert werden können, führen in Deutschland beispielsweise zu Unterschieden in der Lebenserwartung bei Männern um 8,6 und bei Frauen um 4,4 Jahre. Bei den meisten sogenannten ,Volkskrankheiten', wie Diabetes, Atemwegserkrankungen, koronare Herzkrankheiten oder Depressionen, können ebenfalls Unterschiede aufgrund des sozioökonomischen Status beobachtet werden. Dies gilt auch für saisonale Influenza und auch die H1N1-Pandemie im Jahre 2009/2010: Sozioökonomisch benachteiligte Gruppen leiden stärker hinsichtlich Schwere der Erkrankungen, Inzidenz einer symptomatischen Infektion sowie Hospitalisierung und eines tödlichen Verlaufs (Wahrendorf et al. 2020).

Ungleichheiten in Bezug auf Gesundheit können auch hinsichtlich des Geschlechts, Zugehörigkeit zu ethnischen Minderheiten, Pflegebedürftigkeit im Alter sowie hinsichtlich der Gesundheitsrisiken von Lesben, Schwulen, Bisexuellen, trans- und intergeschlechtlichen Personen (LSBTI) beschrieben werden (Pöge et al. 2020). Erst 1990 erfolgte beispielsweise die Streichung von Homosexualität von der Liste der psychischen Erkrankungen durch die WHO, ein wichtiger Schritt für die Anerkennung der sexuellen Vielfalt. Gesundheit war davor also ausschließlich heteronormativ denk- und lebbar. Aus Sabina Misochs (2016) Literaturreview zu Bedürfnissen von homosexuellen Frauen und Männern im dritten und vierten Lebensalter geht deutlich hervor, dass deren Gesundheitszustand schlechter ist als bei gleichaltrigen Heterosexuellen. Als Ursache wird der permanente soziale Stress aufgrund der Normabweichung in einer heteronormativen Gesellschaft angenommen. Sind LSBTI-Personen alt und pflegebedürftig, verheimlichen sie ihre sexuelle Zugehörigkeit nicht selten, um nicht weiterer Diskriminierung ausgesetzt zu sein (Butler 2017; Castro Varela 2016; Misoch 2016).

Die Pflege von Menschen im Alter betrachten Gertrud Backes, Martina Wolfinger und Ludwig Amrhein als "Ausdruck einer geschlechterspezifischen Verknüpfung von Geschlecht, Alter(n) und Pflegebedürftigkeit“" (2008, 132). Über den gesamten Lebenslauf hinweg wird Geschlecht als soziale Klassifikation und als kulturelles Muster kontinuierlich hergestellt 
und hat damit Einfluss sowohl auf die Lebenslage der pflegebedürftigen alten Menschen, als auch auf die Übernahme von Care und Pflegetätigkeiten. Geschlechterungleichheiten, wie z. B., dass Männer häufiger durchgängig in besser bezahlten Jobs arbeiten und Frauen eher - oft durch Phasen der Pflege - unterbrochene Berufskarrieren aufweisen, führen dazu, dass sich Geschlechterhierarchien auch im Alter und in Phasen der Pflegebedürftigkeit fortsetzen. Das hohe Armutsrisiko von alleinstehenden Frauen im hohen Alter, verbunden mit erhöhter Wahrscheinlichkeit chronisch und an mehreren Krankheiten zu leiden und auch eher mit einer demenziellen Erkrankung zu leben, ist ein Ausdruck dieser Verschränkung von Geschlecht mit anderen sozialen Kategorien.

\section{Care-Arbeit war schon vor der Pandemie ungleich verteilt}

Gerade im feministischen Diskurs wird die ungleiche und ungerechte Verteilung von Care-Arbeit schon lange aufgezeigt bzw. ist Teil der Frauenbewegungen von Anbeginn an, da sie eine Wurzel der patriarchalen Ausbeutung von Frauen darstellt. Der Care-Begriff hat sich in den letzten beiden Jahrzehnten im deutschsprachigen Raum gefestigt und Begriffe wie Reproduktionsarbeit oder Hausarbeit in den Hintergrund treten lassen, wenn auch nicht kritiklos (Haug 2013). Cornelia Klinger argumentiert, dass die frühere Terminologie zu stark am herkömmlichen Arbeitsbegriff orientiert ist und das nachvollziehbare Bemühen aufzeigt, „dass das, was Frauen tun, auch Arbeit ist" (Gerhard/Klinger 2013, 268) und plädiert für den Begriff der „Lebenssorge“. Care soll in diesem Beitrag verstanden werden als Ein-sich-um-andere-Kümmern ${ }^{1}$, sowohl privat als auch beruflich, und umfasst die Gesamtheit fürsorglicher Aufgaben wie Beziehungsarbeit, Hausarbeit, Betreuung und Pflege. Wir wollen nicht weiter in eine akademische Debatte über Begrifflichkeiten einsteigen, wollen aber dennoch darauf hinweisen, dass im feministischen Diskurs Einigkeit darüber besteht,

1 Unterschiede gibt es bezüglich der Breite des Care-Begriffs, wer als andere*r, um den*die sich gekümmert wird, gelten kann. Während bei Conradi (2001) eine Care-Beziehung durch mindestens zwei Menschen bestimmt ist, beziehen Fisher und Tronto (1990) die Selbstsorge und Umwelt in ihr Care-Verständnis ein. „On the most general level, we suggest that caring be viewed as a species activity that includes everything that we do to maintain, continue, and repair our 'world' so that we can live in it as well as possible. That world includes our bodies, our selves, and our environment, all of which we seek to interweave in a complex, life-sustaining web" (Fisher/Tronto 1990, 40). 
dass Care-Arbeit v. a. zwischen Frauen und Männern ungleich verteilt ist, was auch als "Gender Care Gap" (Villa 2020, 441f) bezeichnet wird. Aus dem deutschen Gleichstellungsbericht ${ }^{2}$ aus dem Jahr 2017 geht hervor, dass Frauen täglich um 52 Prozent mehr unbezahlte Tätigkeit für andere als Männer leisten. Gender Care Gap und Gender Pay Gap stehen in einem Wechselverhältnis. Um die in einem traditionellen Geschlechterverhältnis (heterosexuelle Paarbeziehung) zugeschriebene Rolle, zu der die Zuständigkeit für die Care-Arbeit in der Familie gehört, ausüben zu können, arbeiten Frauen, v. a., wenn es Kinder gibt, großteils Teilzeit. Bei Erwerbstätigkeit sind Frauen überproportional in sozialen Berufen tätig, die wiederum schlecht bezahlt sind (Villa 2020, 443). Die Zuteilung von Sorgearbeit erfolgt nicht nur nach Geschlecht, sondern auch am Kreuzungspunkt von Klasse und Migration. Mit der Zunahme der Erwerbstätigkeit von Frauen, mit dem bereits erwähnten hohen Anteil an Teilzeitbeschäftigten gegen Ende des 20. Jahrhunderts, kam es zu einer nach wie vor im Steigen begriffenen Externalisierung von Care an Migrant*innen. Wichtiges Beispiel sind die 24-Stunden-Betreuer*innen, in Österreich vorrangig Frauen aus Rumänien und der Slowakei, die sich in prekären Arbeitsverhältnissen in Privathaushalten um pflegebedürftige alte Menschen kümmern (Leiblfinger/Prieler 2018). Helma Lutz und Anna Amelina (2017, 119) sprechen bezüglich der transnationalen Dienstleistungen im Zusammenhang von Care von der „Entstehung neuer, globalisierter sozialer Ungleichheiten“. Die geringe Bewertung von Care-Arbeit, die sich in der Bezahlung und Prekarität niederschlägt, ist Ausdruck der gegebenen hierarchischen Geschlechter-, Klassen- und Migrationsordnung.

\section{Pandemische Ungleichheiten}

\subsection{Eine Frage von Arm und Reich}

Gleich zu Beginn der Pandemie wurde der Blick in diskriminierender Weise auf die Generationen gerichtet. Während die älteren als generell vulnerabel galten - für viele unter ihnen ein Stigma - wurden die jüngeren als verantwortungslos und als Verursacher*innen von Clustern verleumdet. Wir schicken hier voraus, dass alle Altersstufen und Generationen

2 Da Österreich das letzte Mal 2008/2009 an der europaweit durchgeführten Zeitverwendungserhebung teilgenommen hat, liegen keine aktuellen Daten vor. Die Situation ist mit Deutschland aber vergleichbar. 
von der Pandemie betroffen sind, Kindergartenkinder, Schüler*innen, Student*innen, junge Eltern oder ältere und alte Menschen. Jeder Lebensabschnitt hat seine eigenen Sorgen in der Pandemie. Wir schließen uns Anton Amann (2004) an, wenn er meint, dass die ungleiche Verteilung von Vermögen nicht so sehr eine Frage zwischen Jung und Alt, sondern vor allem eine zwischen Arm und Reich ist, und sehen das auch für die Pandemie so.

Stefanie Graefe et al. (2020, 414f) beobachteten im Zuge der ersten Pandemiewelle, dass, wenn sich Ältere in der Öffentlichkeit artikulierten, es zumeist privilegierte Ältere waren, die in einem selbst-heroisierenden Gestus von ihrer Generation einforderten, zu Hause zu bleiben, zugunsten der Jungen und der Wirtschaft. Unter Ausblendung der ungleichen Verteilung von sozialen und materiellen Ressourcen wurden normative Erwartungen von ihnen hervorgebracht, die für weniger privilegierte alte Menschen ohne eigene Immobilie, Terrasse oder Garten andere Konsequenzen haben als für sie. Die Autorinnen kritisieren den Steuerungsmodus der Eigenverantwortlichkeit bei gleichzeitiger Unsichtbarmachung der mangelnden Ressourcen von weniger reicher Alten.

Wir konnten es ja selbst beobachten und bereits wenige Wochen nach der Einführung der ersten Corona-Maßnahmen gab es in der Mannheimer Corona-Studie die ersten zahlenmäßigen Hinweise darauf, dass die soziale Ungerechtigkeit durch Corona zunahm: Im Home-Office, wo die Ansteckungsgefahr geringer ist, waren deutlich mehr Personen mit hohem Bildungsabschluss und gutem Verdienst, während Kurzarbeit und Jobverlust insbesondere Menschen mit geringem Einkommen betrafen (Möhring et al. 2020). Das Infektionsrisiko ist für Menschen in den sogenannten systemrelevanten Berufen ungleich höher. Die extrem schlecht bezahlten, meist aus Südosteuropa stammenden Erntearbeiter*innen oder die Arbeiter*innen in fleischverarbeitenden Fabriken, die in oft unzumutbaren Wohnverhältnissen untergebracht sind, sind ungleich stärker ansteckungsgefährdet als Menschen im Home-Office.

Noch eklatanter ist der Unterschied zwischen armen und reichen Ländern. In den Wochen, in denen wir an diesem Text schreiben, berühren gerade die Bilder aus Brasilien oder Indien. Dort sind Impfungen, Arzneimittel und Sauerstoff knapp und stehen bei weitem nicht für alle zur Verfügung, die sie brauchen. Ärzt*innen und Pflegende stehen vielfach vor dem Zusammenbruch. Zuletzt ist sogar das Brennholz knapp, das benötigt wird, um die vielen Toten zu verbrennen. Weltweit, aber auch innerhalb Europas sind die Mittel, die es braucht, um die Pandemie zu bekämpfen, ungleich verteilt, das betrifft Masken, Schutzkleidung, Sauerstoff bis hin zur Impfung. 


\subsection{Auswirkung des sozioökonomischen Status auf Infektionsrisiko, Schwere der Erkrankung und Höhe der Sterblichkeit}

„Auch beim neuen Coronavirus, das an sich nicht zwischen Menschen unterscheidet, müssen wir davon ausgehen, dass die individuellen gesundheitlichen Folgen stark vom sozialen Status abhängen" (Heisig/König 2020, 38). Wenn wir nun doch zuerst den Fokus auf die körperliche Gesundheit richten, so fällt auf, dass sich die ungleiche Verteilung von Gesundheit in mehreren Parametern zeigt: im ungleichen Infektionsrisiko, in der unterschiedlichen Schwere der Erkrankung und in der höheren Sterblichkeit von sozial Schwächeren, die häufig ethnischen Minderheiten angehören.

Immer neuere empirische Daten weisen darauf hin, dass sozial schwache Gruppen am stärksten durch das neuartige Coronavirus gefährdet sind. So zeigen Daten aus Großbritannien und den USA, dass Angehörige ethnischer Minderheiten sich mit höherer Wahrscheinlichkeit infizieren, einen schwereren Krankheitsverlauf erleiden und ein höheres Sterberisiko haben. Ende April waren 34 Prozent der Covid-19-Erkrankten im Vereinigten Königreich schwarz, asiatisch oder gehörten einer anderen Minderheit an - mehr als doppelt so viele, wie es ihrem Anteil an der Bevölkerung (14 Prozent) entspräche (Li/Heisig 2020, 42).

Es ist bekannt, dass in ärmeren Wohngegenden das Risiko sich mit COVID-19 anzustecken, aber auch daran zu sterben, größer ist als in reicheren Wohngegenden; dies konnte sowohl in UK als auch in den USA gezeigt werden (Morris/Barnes 2020; Wadhera et al. 2020; Whittle/Diaz-Artiles 2020), trifft aber sicher auf alle Länder zu.

Das Konzept der Risikopatient innen macht deutlich, dass die Schwere der COVID-19-Erkrankung mit bestimmten Vorerkrankungen zunimmt:

Neben dem Alter sind Vorerkrankungen wie Übergewicht, Bluthochdruck, Herz-Kreislauf- und Atemwegserkrankungen wichtige Risikofaktoren, und diese sind in Deutschland wie in allen anderen reichen Volkswirtschaften sozial ungleich verteilt. (Heisig/König 2020, 39)

Wie schon erwähnt, ist das Risiko an COVID-19 zu sterben von den sozioökonomischen Verhältnissen abhängig. Die Zusammenhänge sind subtil, wenn auch leider nicht überraschend. So kann für Deutschland gezeigt werden, dass die Belastung durch Industrieabgase (die auch mit Feinstaubbelastung einhergeht) an Wohnorten mit niedrigem Einkommen deutlich höher ist. Und in den USA kann gezeigt werden, dass die COVID-19-Sterb- 
lichkeit bei steigender Feinstaubbelastung höher ist (Heisig/König 2020; Wu et al. 2020).

Mit dem Infektionsrisiko in Zusammenhang stehen auch die Möglichkeiten, zu relevanten Gesundheitsinformationen zu gelangen und Gesundheitsvorsorge zu betreiben. Im Zusammenhang mit COVID-19 stellt die Impfung die wichtigste Präventionsmaßnahme dar, um eine Infektion mit dem Virus zu vermeiden. Selbst wer die Impfung wann erhält, ist sozial geschichtet. In einem Artikel zur Impfreihung des Nachrichtenmagazins profil (2. Mai 2021) wird die Gesundheitsökonomin Maria M. Hofmacher zitiert, die konstatiert, dass schwächere Schichten, zu denen viele Menschen mit Migrationshintergrund zählen, schwerer zu Impfterminen kommen. Um rasch geimpft zu werden, ist Eigeninitiative notwendig und besser Gebildete kommen einfacher zu den dafür notwendigen Informationen. Am Beispiel Wiens wird aufgezeigt, dass sich doppelt so viele Risikopatient*innen aus Nobelbezirken der Innenstadt für eine Impfung angemeldet hatten wie aus dem Arbeiterbezirk Favoriten. Der Weg zur Impfung (Information erhalten, Attest ausstellen lassen, Computeranmeldung) stellt demnach für sozial Schwächere eine wesentlich höhere Hürde dar.

\subsection{Die Folgen der Covid-19-Maßnahmen für kranke und sterbende Menschen}

Aber nicht nur die COVID-19-Erkrankung selbst ist in der Pandemie ungleich verteilt, sondern auch der Zugang zur Gesundheits- und Krankenversorgung insgesamt. Österreich erlebte im Jahr 2020 eine sogenannte Übersterblichkeit. Die Anzahl der Verstorbenen ist von 2019 auf 2020 sprunghaft angestiegen von etwa 83.000 auf etwa 90.000 (Statistik Austria 2021). Auch wenn genaue Auswertungen dazu noch fehlen, so ist bereits jetzt klar, dass nur ein Teil dieser Übersterblichkeit direkt auf das Virus zurückzuführen ist. Eine große Rolle spielt auch die Tatsache, dass in Zeiten der Pandemie der Zugang zu medizinischer Versorgung nicht so gut war wie vor der Pandemie. Die Prioritäten wurden auf die Versorgung von Menschen mit einer COVID-19-Infektion gesetzt, vielfach wurde Menschen mit anderen Erkrankungen der Zugang zur Versorgung verwehrt. Österreich ist da kein Einzelfall.

Befürchtet wird unter anderem, dass die Krebserkrankungen zunehmen werden, weil sich der Zugang zur Vorsorge deutlich verschlechtert hat (Gerechte Gesundheit, 2021).

Für die Sorge am Lebensende zeichnen Abel und Kolleg*innen (2020) ein ähnliches Bild für UK, das auch für andere Regionen zutrifft. Die normale Versorgung am Lebensende wurde in Großbritannien erheblich 
eingeschränkt, so dass nur noch die am schwersten erkrankten Menschen ins Krankenhaus eingeliefert werden. Menschen, die vor der Pandemie möglicherweise eine Beatmungsunterstützung erhalten hätten, kann in der Pandemie der Zugang dazu verweigert werden, weil die entsprechenden Geräte für Corona-Kranke benötigt werden. Oft dürfen Menschen, die zum Sterben ins Krankenhaus eingeliefert werden, keine Besuche von Angehörigen empfangen und die Zahl der Teilnehmer*innen an Beerdigungen ist begrenzt. Die Wahl des bevorzugten Sterbeortes kann verweigert werden, ob zu Hause oder im Krankenhaus.

Wir beziehen uns auf ein ganzheitliches Menschenbild, wie es Palliative Care zugrunde liegt. Auch und gerade in der Pandemie ist es ratsam, nicht hinter die gewonnenen Erkenntnisse zurückzufallen. Es lohnt sich, sich das Konzept von Total Pain in Erinnerung zu rufen und das Menschenbild, das dahintersteht. Dieses Menschenbild geht davon aus, dass wir körperliche, seelische, soziale und spirituelle Wesen sind, und Gesundheit wie auch Krankheit durch alle diese Ebenen beeinflusst werden. So sind sowohl soziale als auch psychische und spirituelle Bedürfnisse ebenso wichtig wie physisch-körperliche. Der Fokus in der Krise liegt auf der virologischen Gesundheit - wir fragen uns: Wieso gelingt es in der Krise nicht, an die bisherigen Erkenntnisse anzuknüpfen? (Rösler et al. 2020)

\subsection{Die Situation von Menschen mit Vergesslichkeit - zwischen Schutz und Selbstbestimmtheit}

Gerade für Menschen mit kognitiven Einschränkungen und mit Vergesslichkeit sind die Maßnahmen der Corona-Pandemie besonders schwer nachvollziehbar. Oft können sie sich weder die Maßnahmen noch die Begründung dafür merken. Gelingende Kommunikation bildet die Grundlage für soziale Teilhabe und damit für ganzheitliche Gesundheit. Ob ein Leben mit Demenz lebenswert ist, hängt entschieden davon ab, wie sich die Umwelt gegenüber Menschen mit Demenz verhält. Wenn nun die Umwelt auf zuerst einen, später auf zwei Meter Distanz geht und eine Maske trägt, was mag das für Menschen mit Demenz bedeuten? Welche Schlüsse ziehen sie daraus? Kann Kommunikation so gelingen? Auch stand vor allem zu Beginn der Pandemie im ersten harten Lockdown die Vorstellung im Vordergrund, dass Menschen am sichersten sind, wenn sie das Haus nicht verlassen. Dies wurde in Österreich auch dadurch verstärkt, dass sich die Politik in Widersprüche verwickelt hat, was die Gründe, für die es erlaubt war, das Haus zu verlassen, betrifft. Sie waren also zunächst nicht klar und wechselten auch rasch. Pflegende Angehörige von Menschen mit 
Demenz - in Österreich mehrheitlich Frauen - kamen dadurch in die Situation, den zu Pflegenden nahebringen zu wollen oder sollen, dass sie das Haus nicht verlassen dürfen. Dies ist aufgrund der Vergesslichkeit und des häufig großen Bewegungsbedürfnisses von Menschen mit Demenz eine unlösbare Aufgabe. Es überrascht nicht, dass Erzählungen von Gewalt in der Pflege zunahmen. Professionell und ehrenamtlich Beratende und Betreuende standen vor der Frage, ob und wie sie sich beratend positionieren sollen: Ging es vor allem um Sicherheit, das heißt darum, Angehörige dabei zu unterstützen, wie sie die Menschen mit Demenz daran hindern könnten, das Haus zu verlassen, oder stand die Selbstbestimmtheit der Menschen mit Demenz im Vordergrund und damit das Risiko, dass sie sich bei einem Spaziergang ansteckten, weil sie die Hygienemaßnahmen nicht einhalten konnten (Rösler et al. 2020)? In einer von den Autorinnen während der Pandemie durchgeführten Studie wurden in Interviews von Menschen mit Demenz wie auch ihren Angehörigen ebenfalls Belastungen angesprochen, aber auch positive Aspekte erwähnt. Wenn die noch berufstätigen pflegenden Angehörigen in Home-Office waren, erlebten dies die Personen mit Demenz durchaus als angenehm. Auch das Erlernen von Video-Meetings war für manche etwas Spannendes. Im Großen und Ganzen wurden jedoch mehr die Restriktionen durch die Pandemie beklagt, wie z. B. das Schließen der Tageszentren im ersten Lockdown. Die Hygienemaßnahmen führten vereinzelt zu Überforderungen beim Einkauf, sodass manche nicht mehr alleine wie vor der Pandemie einkaufen gingen, was zu Verlusten der Eigenständigkeit und des sozialen Aktionsradius führte (Pichler et al. 2021).

Auch in den Pflegeheimen spitzte sich das Dilemma zwischen Sicherheit und Selbstbestimmung zu: Angesichts der Tatsache, dass im ersten Jahr der Pandemie so viele Bewohner*innen von Pflegeheimen an COVID-19 verstarben, wurden drastische Maßnahmen gesetzt, um Ausbrüche der Infektion in den Pflegeheimen zu verhindern. Wochen- bis monatelange Besuchsverbote waren die Folge. Dieser exklusive Blick auf die virologische Gesundheit führte zu großem Leid der hochbetagten Menschen, denn auch Einsamkeit tut weh. Durch die Geschlechterbrille gesehen, ist es ein Dilemma, das vielfach Frauen betrifft, leben und arbeiten doch wesentlich mehr Frauen in einem Pflegeheim (Reitinger et al. 2016), wie auch mehr Frauen von Demenz betroffen sind. 


\subsection{Die Konsequenzen der Pandemie für Pflege- und Betreuungspersonen}

Die Belastungen in der Krise betreffen Care-Arbeiter*innen ungleich stärker: Besonders betroffen vom erhöhten Infektionsrisiko ist die Pflege. Denn Pflege erfordert den physischen Kontakt mit oder die Nähe zu den Betroffenen, dadurch wird sie hochgefährlich. Es handelt sich dabei um einen großteils von Frauen ausgeübten Beruf, 92,2 Prozent der formellen Pflege- und Betreuungspersonen sind in Österreich weiblich (Statistik Austria 2019). Es reicht nicht aus, dass dieses erhöhte Risiko den Pflegenden durch einen Applaus um 18:00 abgegolten wurde. Die Gefährlichkeit der Pflege in Zeiten der Pandemie schränkt die Fähigkeit, Pflege in der erforderlichen Qualität zu leisten, drastisch ein (Fine/Tronto 2020). Zu Recht wird die Frage laut: „Who or what cares for care“ (Branicki 2020)? Oder auch: Wer hilft den Helfenden? Die Berichte darüber, dass Pflegende gegen ihren Willen auf Corona-Stationen versetzt werden und dort in bunt zusammengewürfelten, einander fremden Teams in Schutzanzügen ihren Dienst verrichten, nötigen Respekt ab. Dass gleichzeitig und quasi reflexartig - wie vielfach berichtet wird - Besprechungszeiten, wie zum Beispiel Dienstübergaben, eingespart werden, um mehr Zeit für die direkte Pflege zu haben, ist ein bedauerlicher Irrtum: gerade in Krisen bringen Zeiten der Reflexion ein wenig und dringend benötigte Entlastung.

Wie bereits ausgeführt, etabliert sich für die Sorge von Pflegebedürftigen zu Hause zunehmend die 24-Stunden-Betreuung als Modell, schon in Zeiten ohne Pandemie ein fragiles Modell, das auf Einkommensungleichheiten zwischen reicheren und ärmeren Ländern beruht. Die symbolische Anerkennung von Sorgearbeit zu Beginn der Pandemie konnte nicht darüber hinwegtäuschen, dass die Maßnahmen zu einer Zunahme des Prekariats der 24-Stunden-Betreuerinnen führten. Insbesondere die Ausgangsund Reisebeschränkungen stellten emotionale und physische Belastungen dar, Zeiten der Quarantäne und Einreiseverbote führten zu finanziellen Einbußen und Unsicherheiten (Leiblfinger et al. 2021).

Auch für pflegende Angehörige hat die Pandemie gravierende Auswirkungen. In Österreich sind 73 Prozent der pflegenden Angehörigen Frauen (Nagl-Cupal et al. 2018), sodass diese Belastung mehrheitlich Frauen betrifft. Die Ergebnisse einer von der Volkshilfe (2021) im Frühjahr 2021 in Österreich durchgeführten Umfrage zeigen, dass für mehr als die Hälfte der Angehörigen die Betreuung und Pflege aufwendiger geworden ist, die Lebensqualität deutlich gesunken ist und es v. a. zu wenig Entlastungsmöglichkeiten gab und gibt. 67 Prozent der Befragten sprechen von körperlichen oder emotionalen Herausforderungen im Rahmen der Pflege während der Corona-Krise, viele nennen auch finanzielle Probleme. 


\subsection{Geschlechter(un)gerechtigkeit in der Care-Arbeit}

Zwei Szenarien sind prinzipiell denkbar, das erste: Corona könnte die Geschlechtergerechtigkeit verbessern. Die Überlegungen, die dieses Szenario unterstützen, sind folgende: die systemrelevanten Berufe werden mehrheitlich von Frauen ausgeübt; Frauen müssen daher auch in der Krise ihrer Erwerbstätigkeit nachgehen und gleichzeitig könnten Väter im Home-Office mehr Zeit für ihre Kinder haben. Auch sind Männer öfter selbstständig als Frauen und es hätte sein können, dass sie daher in der Krise ihr Unternehmen verlieren, weniger Zeit für Erwerbsarbeit und mehr Zeit für die Kinderbetreuung aufbringen. Das zweite Szenario geht davon aus, dass Mütter zusätzlich zur Berufstätigkeit und zur reproduktiven Tätigkeit im Haushalt nun auch die Sorge für den Unterricht ihrer Kinder zu Hause übernehmen. Die verfügbaren Zahlen weisen darauf hin, dass das Szenario der abnehmenden Gendergerechtigkeit eher zutrifft. Gleich in den ersten Wochen der Pandemie wies die Mannheimer Corona-Studie nach, dass die Wahrscheinlichkeit, nach einem Lockdown die Arbeit zu verlieren, für Frauen höher war als für Männer. Mütter übernehmen im Lockdown mehr Sorgearbeit für die Kinder als Väter, gleichzeitig bleibt die Verteilung der Hausarbeit gleich (Hipp/Bünning 2021). Auch die Sorgearbeit im Bereich der Angehörigenpflege hat im ersten Lockdown zugenommen: ausfallende 24-Stunden-Betreuerinnen, geschlossene Tageszentren und Sorge vor Ansteckung durch professionelle Betreuungspersonen in der häuslichen Pflege wurden durch die überwiegend weiblichen pflegenden Angehörigen kompensiert. Damit betrifft die Corona-Krise Frauen doppelt, durch weniger Erwerbseinkommen und mehr Sorgearbeit (Kohlrausch/Zucco 2020). Diese Mehrfachbeanspruchung durch die Care-Arbeit zu Hause ist ein wesentlicher Belastungsfaktor mit Folgen für die psychische und physische Gesundheit von Frauen (Büro für Frauengesundheit und Gesundheitsziele der Stadt Wien 2020).

„Any boat in a storm“: Es scheint wohl so zu sein, und jede*r kennt das aus eigenem Erleben: in Krisenzeiten greifen wir schnell auf Altbewährtes zurück (Allmendinger 2020). Die Pandemie hat unseren Alltag, wesentliche Gesellschaftsbereiche und -ordnungen durcheinandergebracht und uns alle verunsichert. Altbewährtes gibt Sicherheit, Reibungsverluste und aufwendige Aushandlungsprozesse fallen durch den Rekurs auf eingeübte Traditionen weg. So ist es auch in der Corona-Krise: die zunehmenden Belastungen lassen weniger Zeit für Aushandlungsprozesse zwischen den Geschlechtern, die Verunsicherung lässt Paare und Familien auf traditionelle Rollenteilungen zurückgreifen. 
Die Auswirkungen dieser Retraditionalisierung sind vielfältig. Traditionelle Familienbilder sind heteronormativ geprägt und werden in der Krise wieder dominanter. Daher macht u. a. der Lesben- und Schwulenverband Deutschlands darauf aufmerksam, dass und wie die politischen Maßnahmen im Zusammenhang mit Corona diskriminierend wirken:

Die Idee der heilen Familie ignoriert sowohl das massive Vorkommen von häuslicher Gewalt als auch die mitunter gravierenden Diskriminierungserfahrungen, die LSBTI in ihren Herkunftsfamilien machen müssen und daher mit diesen gebrochen haben. Freundschaften als Wahl- und Ersatzfamilie sind daher für LSBTI essenziell und überlebenswichtig. Ihnen soll jetzt ein gemeinsames Weihnachten verboten werden. Das verstärkt die soziale Isolation von LSBTI. (LSVD 2020)

Dies ist für ältere LSBTI besonders brisant, als diese oft keine jüngeren familiären Angehörigen haben, die sich um sie kümmern und sie häufiger alleine leben. Vor allem diejenigen ohne Partner*innen oder Mitbewohner*innen sind daher eher dem Risiko von Einsamkeit und Isolation ausgesetzt. Mehrere Freund*innen zu treffen und einander in Gruppen zu sehen war lange Zeit nicht möglich (LSVD 2020).

Die Einschränkung auf Kontakte zwischen Personen, die „im selben Haushalt leben", wurde in Österreich erst im Laufe der Zeit erweitert zu Kontakten mit „engen Bezugspersonen“. Dieser Fokus führt einerseits, wie beschrieben, zu weniger oder schwierigerem Kontakt für Menschen, die alleine leben. Andererseits nahm durch diese Verdichtung und das Zusammenleben auf engem Raum die Gewalt in Familien und Gewalt an Frauen zu. Denn obwohl weniger Frauen den Schutz von Frauenhäusern aufsuchten, stieg die Anzahl der telefonischen Beratung während der LockdownZeiten erheblich an. Vor allem psychische und ökonomische Gewalt und die Dauer der Beratungsgespräche haben zugenommen (red.wien.ORF.at, 2021).

\section{Plädoyer für Care-Ethik in der Krise}

Im Krisenmanagement wird und wurde vielfach eine maskuline und martialische Sprache verwendet. Ein Höhepunkt wurde erreicht, als Frankreichs Staatspräsident im März 2020 die Verschärfung der Corona-Maßnahmen begründete mit „Nous sommes en guerre“ („Wir sind im Krieg“). Branicki (2020) unterscheidet zwischen dem derzeit stattfindenden rationalen Krisenmanagement und einem wünschenswerten feministischen Krisenmanagement. Während rationales Krisenmanagement ein Abwägen 
von Kosten und Nutzen betont und darauf abzielt, zur alten Normalität zurückzukehren, betont ein feministisches Krisenmanagement die relationale Logik von Zusammenleben auch in der Krise. Daher sind die Stärkung und Ausweitung von Beziehungen, das füreinander Sorgen und einander Unterstützen, Wege, über die Möglichkeiten zur sozialen Transformation führen.

Insofern ist ein Hauptunterschied in den Zugangsweisen die Perspektive der Zeitlichkeit: Rationales Krisenmanagement geht davon aus, dass eine Krise zeitlich begrenzt und sozial spezifisch ist. Im Gegensatz dazu sieht feministisches Krisenmanagement Krisen als andauernde und einander überlappende Phänomene, die miteinander zusammenhängen und denen vor allem durch die Stärkung von Beziehungen und Netzwerken begegnet werden kann. So formuliert Barbara Thiessen (2021): „Wenn zur Krise noch eine Pandemie dazukommt".

In vielfacher Weise macht die Krise deutlich, wie sehr wir aufeinander verwiesen sind. Das sogenannte Social Distancing ist ja zunächst ein Physical Distancing, das in vielen Situationen, wie zum Beispiel im Pflegeheim, bedauerlicher Weise zu sozialer Distanz führt. Es macht uns deutlich, welche Rolle Beziehung und Nähe für unser psychisches, soziales, spirituelles, aber auch körperliches Wohlbefinden spielen. Auch hat sich herausgestellt, dass vorrangig jene Berufe relevant für ,das System' sind, in denen es um fürsorgliche Aufgaben geht: Pflegende, Ärzt*innen, Apotheker*innen, Rettungsdienste, Feuerwehr, Pädagog*innen und jene Menschen, die uns dazu verhelfen, dass wir unsere Grundbedürfnisse nach Essen und Hygiene abdecken können (Schlegel 2020). Dass es eine Ethik braucht, die von Care ausgeht, ist ja nicht ein Gedanke, der erst in der Pandemie entstanden ist. Fine und Tronto (2020) sprechen von „the hidden truths about caring revealed by the COVID-19 pandemic”. COVID-19 und der Umgang damit machen die Bedeutung der Ethik der Fürsorglichkeit umso deutlicher.

Neben allem Leid, das die Pandemie mit sich bringt, birgt sie doch eine Chance für die Gesellschaft zu lernen, gerade in Bezug auf die gleiche und gerechte Verteilung von Care und Gesundheit (Care.Macht.Mehr 2021). Den Apellen, dass nach der Pandemie nicht vor der Pandemie werden soll, können wir uns hier nur anschließen. 


\section{Literatur}

Abel, Julian/Kellehear, Allan/Millington Sanders, Catherine/Taubert, Mark/ Kingston, Helen: Advance care planning re-imagined: a needed shift for COVID times and beyond, in: Palliative Care \& Social Practice 14 (2020) 1-8. DOI: 10.1177/2632352420934491.

Allmendinger, Jutta: Zurück in alte Rollen Corona bedroht die Geschlechtergerechtigkeit, in: WZB Mitteilungen 168 (2020) 45-47.

Amann, Anton: Die großen Alterslügen. Generationenkrieg - Pflegechaos - Fortschrittsbremse? Wien: Böhlau 2004.

Backes, Gertrud M./Wolfinger, Martina/Amrhein, Ludwig: Geschlechterungleichheiten in der Pflege, in: Bauer, Ulrich/Büscher, Andreas: Soziale Ungleichheit und Pflege, Wiesbaden: VS Verlag für Sozialwissenschaften 2008, 132-153.

Bolte, Gabriele/Lahn, Ulrike: Geschlecht in der Public-Health-Forschung zu gesundheitlichen Ungleichheiten: Potenziale und Begrenzungen des Intersektionalitätsansatzes. GENDER - Zeitschrift für Geschlecht, Kultur und Gesellschaft 7/2 (2015), 51-67.

Branicki, Layla: COVID-19, ethics of care and feminist crisis management, in: Gender, Work \& Organization 27 (2020) 872-883. DOI: 10.1111/gwao.12491.

Büro für Frauengesundheit und Gesundheitsziele der Stadt Wien (Hg.): Frauengesundheit und Corona. Sammelband des Wiener Programms für Frauengesundheit. Schriftenreihe Frauen*Gesundheit*Wien 1 (2020), in: https://www. wien.gv.at/gesundheit/beratung-vorsorge/frauen/frauengesundheit/pdf/frauenge sundheit-corona.pdf [04.06.2021].

Butler, Sandra S.: Older lesbians' experiences with home care: Varying levels of disclosure and discrimination, in: Journal of Gay \& Lesbian 29/4 (2017) 378398. DOI: 10.1080/10538720.2017.1365673.

Care.Macht.Mehr: Großputz! Care nach Corona neu gestalten (2021), in: https://ca re-macht-mehr.com/ [15.06.2021].

Castro Varela, Maria do Mar: Altern Andere anders? Queere Reflexionen, in: Lottmann, Ralf/Lautmann, Rüdiger/Castro Varela, Maria do Mar: Homosexualität_en und Alter(n). Ergebnisse aus Forschung und Praxis. Wiesbaden: Springer VS 2016, 51-67.

Conradi, Elisabeth: Take Care. Grundlagen einer Ethik der Achtsamkeit. Frankfurt, New York: Campus Verlag 2001.

Crenshaw, Kimberlé W. (2013): Die Intersektion von „Rasse“ und Geschlecht demarginalisieren: Eine Schwarze feministische Kritik am Antidiskriminierungsrecht, der feministischen Theorie und der antirassistischen Politik, in: Lutz, Helma/Herrera Vivar, Maria Teresa/Supik, Linda: Fokus Intersektionalität. Bewegungen und Verortungen eines vielschichtigen Konzeptes, Wiesbaden: VS Verlag für Sozialwissenschaften 2013, 35-58.

Fine, Michael/Tronto, Joan: Care goes viral: care theory and research confront the global COVID-19 pandemic, in: International Journal of Care and Caring 4/3 (2020) 301-309. DOI: 10.1332/239788220X15924188322978. 
Fisher, Berenice/Tronto, Joan: Toward a feminist theory of caring, in: Abel E. K./ Nelson M.K. (Eds.): Circles of care: Work and identity in women's lives. New York: State University of New York 1990, 35-62.

Gerhard, Ute/Klinger, Cornelia: Im Gespräch - Care/Fürsorgliche Praxis und Lebenssorge, in: Feministische Studien 2/13, Stuttgart: Lucius \& Lucius 2013, 267-277.

Gerechte Gesundheit: Versorgung von Krebspatienten hochgefährdet (22.04.2021), in: https:/www.gerechte-gesundheit.de/news/detail/versorgung-von-krebspatien ten-hochgefaehrdet.html [18.06.2021].

Graefe, Stefanie/Haubner, Tine/van Dyk, Silke: „Was schulden uns die Alten?“ Isolierung, Responsibilisierung und (De-)Aktivierung in der Corona-Krise, in: Leviathan 48/3 (2020) 407-432. https://doi.org/10.5771/0340-0425-2020-3-407.

Haug, Frigga: Das Care-Syndrom: ohne Geschichte hat die Frauenbewegung keine Perspektive, in: Widerspruch: Beiträge zu sozialistischer Politik 32/62 (2013.) http://doi.org/10.5169/seals-651848.

Heisig, Jan Paul/König, Christian: Das Virus ist nicht demokratisch. Soziale Ungleichheit prägt den Verlauf der Infektion, in: WZB Mitteilungen 168 (2020) 39-41.

Hipp, Lena/Bünning, Mareike: Parenthood as a driver of increased gender inequality during COVID-19? Exploratory evidence from Germany, in: European Societies 23/1 (2021) 658-673. https://doi.org/10.1080/14616696.2020.1833229.

Kohlrausch, Bettina/Zucco, Aline: Die Corona Krise trifft Frauen doppelt. Weniger Erwerbseinkommen und mehr Sorgearbeit, in: Policy Brief WSI 40 (2020). https://www.wsi.de/de/faust-detail.htm?sync_id=HBS-007676.

Kümpers, Susanne/Alisch, Monika: Ungleichheiten des Alter(n)s in sozialräumlicher Perspektive, in: Bleck, Christian/van Rießen, Christian/Knopp, Reinhold: Alter und Pflege im Sozialraum. Theoretische Erwartungen und empirische Bewertungen. Wiesbaden: Springer VS 2018, 53-68.

Leiblfinger, Michael/Prieler, Veronika/Schwiter, Karin/Steiner, Jennifer/Benazha Aranke, Vanessa/Lutz, Helma: Auswirkungen der Maßnahmen zur Eindämmung der COVID-19-Pandemie auf Live-in-Betreuer*innen, in: Aulenbacher Brigitte/Lutz Helma/Schwiter Karin: Gute Sorge ohne gute Arbeit? Weinheim: Beltz Juventa 2021, 92-103.

Leiblfinger, Michael/Prieler, Veronika: Elf Jahre 24-Stunden-Betreuung in Österreich. Eine Policy- und Regime-Analyse. Linzer Beiträge zu Wirtschaft-EthikGesellschaft 9, Linz: Katholische Privat-Universität Linz 2018.

Li, Jianghong/Heisig, Jan Paul: Der Teufelskreis von Infektion und Ungleichheit. Corona hat soziale Ursachen und Folgen, in: WZB Mitteilungen 168 (2020) 42-44.

LSVD: Corona: Auswirkungen auf Lesben, Schwule, Bisexuelle, trans- und intergeschlechtliche Menschen. Wie sich Covid19 auf das Leben von LSBTI auswirkt (2020), in: https://www.lsvd.de/de/ct/2067-Corona-Auswirkungen-auf-Lesben-Sc hwule-Bisexuelle-trans-und-intergeschlechtliche-Menschen [23.05.2021]. 
Lutz, Helma/Amelina, Anna: Gender, Migration, Transnationalisierung. Bielefeld: transkript Verlag 2017.

Lutz, Helma/Herrera Vivar, Maria Teresa/Supik, Linda: Fokus Intersektionalität - eine Einleitung, in: Lutz, Helma/Herrera Vivar, Maria Teresa/Supik, Linda: Fokus Intersektionalität. Bewegungen und Verortungen eines vielschichtigen Konzeptes. Wiesbaden: VS Verlag für Sozialwissenschaften 2013, 9-31.

Misoch, Sabina: „Lesbian, gay \& grey“. Besondere Bedürfnisse von homosexuellen Frauen und Männern im dritten und vierten Lebensalter, in: Zeitschrift für Gerontologie und Geriatrie (2016). DOI 10.1007/s00391-016-1030-4.

Möhring, Katja/Naumann, Elias/Reifenscheid, Maximiliane/Blom, Annelies G./ Wenz, Alexander/Rettig, Tobias/Lehrer, Roni/Krieger, Ulrich/Juhl, Sebastian/Friedel, Sabine/Fikel, Marina/Cornesse, Carina: Die Mannheimer CoronaStudie: Schwerpunktbericht zu Erwerbstätigkeit und Kinderbetreuung (2020), in: https:/www.uni-mannheim.de/gip/corona-studie/ [29.04.2021].

Morris, Chris/Barnes, Oliver: Coronavirus: Which regions have been worst hit? (2020), in: https://www.bbc.com/news/52282844 [13.05.2021].

Nagl-Cupal, Martin/Kolland, Franz/Zartler, Ulrike/Mayer, Hanna/Bittner, Marc/ Koller, Martina/Parisot, Viktoria/Stöhr, Doreen: Angehörigenpflege in Österreich. Einsicht in die Situation pflegender Angehöriger und in die Entwicklung informeller Pflegenetzwerke, in: Bundesministerium für Arbeit, Soziales, Gesundheit und Konsumentenschutz, Wien: Universität Wien 2018.

Pichler, Barbara/Egger, Barbara/Heimerl, Katharina/Reitinger, Elisabeth: Menschen mit Demenz und (assistive) Technologie. Perspektive der Betroffenen und ihrer informellen und formellen Betreuungs- und Pflegepersonen im häuslichen Settings, in: Bundesministerium für Soziales, Gesundheit, Pflege und Konsumentenschutz, Wien 2021 [im Erscheinen].

Pöge, Kathleen/Dennert, Gabriele/Koppe, Uwe/Güldenring, Annette/Matthigack Ev B./Rommel, Alexander: Die gesundheitliche Lage von lesbischen, schwulen, bisexuellen sowie trans- und intergeschlechtlichen Menschen, in: Journal of Health Monitoring 5/1 (2020). DOI 10.25646/6448.

Profil: Analyse. Geimpft im Homeoffice? Profil 52/18 (2021) 22-23.

red.wien.ORF.at: Weniger Schutzsuchende in Frauenhäusern (26.02.2021), in: https://wien.orf.at/stories/3091546/ [23.05.2021].

Reitinger, Elisabeth/Lehner, Erich/Pichler, Barbara/Heimerl, Katharina: „Doing Gender" im Altenpflegeheim: Perspektiven von Mitarbeitenden und Führungskräften, in: Zeitschrift für Gerontologie und Geriatrie 49/8 (2016) 700-705. DOI 10.1007/s00391-016-1147-5.

Rösler, Petra/Heimerl, Katharina/Dressel, Gert/Bachinger, Nicole/Schönborn, Raphael/Wegleitner, Klaus: Care trotz Corona mit und für Menschen im Alter. Ein Nachdenk- und Diskussionspapier, Wien: kardinal könig haus 2020.

Schlegel Lena: Care-Ethik und Corona: Eine Perspektive der (Für-)Sorge (15.04.2020), in: https://uni-tuebingen.de/einrichtungen/zentrale-einrichtun gen/internationales-zentrum-fuer-ethik-in-den-wissenschaften/publikationen/blo g-bedenkzeiten/weitere-blog-artikel/care-ethik-und-corona-eine-perspektive-der-f uer-sorge/ [29.04.2021]. 
Statistik Austria: Betreuungs- und Pflegedienste (2019), in: http://www.statistik.at/ web_de/statistiken/menschen_und_gesellschaft/soziales/sozialleistungen_auf_la ndesebene/betreuungs_und_pflegedienste/index.html [04.06.2021].

Statistik Austria: Sterbefälle des Jahres 2020 im Vergleich zum Durchschnitt der Jahre 2015 bis 2019 nach Kalenderwoche und Geschlecht (2021), in: https://ww w.statistik.at/web_de/presse/125167.html [13.05.2021].

Stiglitz Joseph: The Price of Inequality: How Today's Divided Society Endangers Our Future, New York: Norton 2012.

Stiglitz Joseph: The great divide: Unequal Societies and What We Can Do About Them, New York: Penguin 2016.

Stronegger Willibald J.: Gesundheitliche Ungleichheit -Verhalten oder Verhältnisse? Sozial-epidemiologische Modelle und Evidenz, in: FGÖ: Soziale Ungleichheit und Gesundheit. Tagungsband der 9. Österreichischen Präventionstagung 2007, in: https://fgoe.org/sites/fgoe.org/files/2017-10/2009-08-28\%204.pdf [21.06.2021].

Thiessen Barbara: Großputz! Care nach Corona neu gestalten (20.01.2021), in: https:/www.fes.de/themenportal-gender-jugend-senioren/gender-matters/gender -blog/beitrag-lesen/grossputz-care-nach-corona-neu-gestalten [29.04.2021].

Villa, Paula-Irene: Corona-Krise meets Care-Krise - Ist das systemrelevant?, in: Leviathan 3 (2020), 433-450.

Volkshilfe: Österreichweite Umfrage von pflegenden Angehörigen. Pressekonferenz 11.05.2021, in: https://www.volkshilfe.at/fileadmin/user_upload/Media_L ibrary/Bilder/Bilder_nach_Themen/Pflege/PK_Ergebnisse_Umfrage_2021.pdf [04.06.2021].

Wadhera, Rishi K./Wadhera, Priya/Gaba, Prakriti/Figueroa, Jose F./Joynt Maddox, Karen E./Yeh, Robert W/Shen, Changyu: Variation in COVID-19 Hospitalizations and Deaths Across New York City Boroughs. Chicago: JAMA (2020). doi:10.1001/jama.2020.7197.

Wahrendorf, Morten/Knöchelmann, Anja/von dem Knesebeck, Olaf/Vonneilich, Nico/Bolte, Gabriele/Lehmann, Frank/Schmidt, Maike J./Butler, Jeffrey/Schmidt, Felicitas/Böhm, Claudia/Lunau, Thorsten/Dragano, Nico: Verschärfen COVID-19 Pandemie und Infektionsschutzmaßnahmen die gesundheitlichen Ungleichheiten? [Hintergrundpapier, AG Indirekte Gesundheitsfolgen von Maßnahmen des Infektionsschutzes des Kompetenznetzes Public Health zu COVID-19] (2020), in: https://www.public-health-covid19.de/images/ 2020/Ergebnisse/Hintergrundpapier_SozUngl_COVID19_final.pdf [03.06.2021].

Whittle, Richard S./Diaz-Artiles, Ana: An ecological study of socioeconomic predictors in detection of COVID-19 cases across neighborhoods in New York City. BMC Medicine 18/271 (2020). https://doi.org/10.1186/s12916-020-01731-6.

WHO Europe: Ottawa Charter für Gesundheitsförderung (1986), in: https:/fgoe.or g/glossar/ottawa_charta [04.05.2021].

WHO Europe: Health inequity and the effects of COVID-19: assessing, responding to and mitigating the socioeconomic impact on health to build a better future. Copenhagen: WHO Regional Office for Europe 2020. 
Wu, Xiao/Nethery, Rachel C./Sabath, Benjamin M./Braun, Danielle/Dominici, Francesca: Exposure to Air Pollution and COVID-19 Mortality in the United States: A Nationwide Cross-Sectional Study (2020), in: https://www.medrxiv.org/content/10.1101/2020.04.05.20054502v1 [28.05.2020]. 
